\title{
Cloud-Based Global Online Marketplaces Review on Trust and Security
}

https://doi.org/10.3991/ijim.v13i04.10523

\author{
Osman Ghazali ( ${ }^{\bowtie}$,Chun Yang Leow, Shahzad Qaiser, Nanthini Pattabiraman, \\ Sathiyaroobaa Vasuthevan, Eman Mohamed Abdusalam, Mustafa M. Barakat \\ Universiti Utara Malaysia, UUM Sintok, Kedah, Malaysia \\ kumar1612@yahoo.com
}

\begin{abstract}
Customer disposition to data, nature of the information on site, protection concerns, trust, security concerns, and the notoriety of organization efficaciously affect the trust of Internet shoppers in the site. Two noteworthy and basic issues for e-commerce sites and consumers are trust as well as security. A belief that someone is good and honest and will not harm you, or something is safe and reliable is called trust; while security is an attempt to safeguard the data from unauthorized access. Information security is a vital management as well as technical requirement over the internet for effective and secure payment transaction activities. The safety of e-commerce resources from use, destruction, unauthorized access and alteration is known as E-commerce security so there is an urgent need to study its dimensions such as authenticity, integrity, availability, privacy, confidentiality and non-repudiation. This paper reports a review of four popular online marketplaces which are Alibaba, Amazon, eBay andTaoBao as case study on two main criteria namely building trust among users and ensuring security on the platform. Furthermore, we discuss the methods being used by each online marketplace to build trust and their unique way ofimproving the security. Finally, different ways of building trust and technique to ensure the security is presented in a tabular form for each online marketplace.
\end{abstract}

Keywords - Building trust, marketplaces, trust, risk, behavior, security, ecommerce.

\section{Introduction}

The number of online marketplaces have been increased over the last decade tremendously. Today, people have access to such marketplaces even in remote areas where there was no access to such facilities before. Online marketplaces have reduced transaction costs giving an opportunity to small scale dealers to run their businesses which encourages the exchange of goods and services which was not possible before. It is very important for the dealers to maintain and build the trust and keep their marketplaces and practices secure enough for the customers so that they keep using the service with safety [1].These days the Internet is widely used for correspondence in business activities and day to day life of the customers. Specialists are persuaded that 
the Internet is the best approach for marketplaces due to its actual web-based business potential [2].

There has been a great increase in number of online commercial centers in the last decade, ranging from Amazon, eBay to the Airbnb and Uber. Two most important factors for such online markets without which they cannot sustain are trust and security. Customers uses marketplace with an assumption that the vendors precisely depict items or benefits and are satisfying exchanges as promised. While on the other hand, vendors serve customers by assuming that they will make payments on time and will abide by the terms and conditions agreed for the service. Notoriety frameworks as well as planning decisions taken by the online marketplaces are responsible to ensure trust among dealers and customers[13]. In ecommerce, it is very important to maintain good relationship with customers otherwise they will move to another option of their choice in a friction of time [15].

Security in e-commerce is particularly ensured for the important matters that directly influence it i.e. data integrity, availability of the system and others that can be found under Information Security framework. As per the report by Forrester, Business to Consumer or simply (B-to-C) Internet commerce is steadily growing on year-onyear basis by $19 \%$. The report further mentioned that B-to-C internet commerce is adapted mode for shopping by a lot of customers [2]. As argued by numerous scholars, successful commerce needs a precondition which is a trust to encourage the customers to purchase from the seller [3], [4] and [5].

Safeguarding the consumer's data to make sure that it is private as well as confidential saves customers from any possibility of a fraud. Customer's tends to use the marketplace that ensures them about the privacy as well as confidentiality of their private data. They also want to control their data freely in a sensible manner. Consumer is more flexible for taking any risk with organizations whom they trust. The probability of the risk is way high in e-commerce because of insignificant physical interactions or unawareness and proximity. So, in order to predict whether the consumer will engage or not in an ecommerce activity, their trust in e-commerce (marketplace) must be studied [14].

The foundation of trusted transaction processes proves to be a key indicator of success in Internet business as dealers needs to create a feasible environment for the customers where they can be comfortable as well as confident while making any potential transactions [6]. Trust has a great role and impact to determine the customer's behavior with merchants [7] and in environments that are uncertain i.e. Internet based environments (EC) [8].From the last several years, the ecommerce growth has outpaced every growth in the retailers operating offline. For example, there was $1.3 \%$ increase witnessed in retail sales in the US in first quarter of 2015, while on the other hand, the ecommerce grew by $3.7 \%$ [12].

Ecommerce (online marketplaces) is mostly seen as one of the most important application in open worldwide computer network infrastructures known as Internet. Currently, global computer networks offer official access to huge number of services as well as functions. As shown in Table I, Alibaba, Amazon, eBay andTaoBao are the few most popular online marketplaces in the world. Although they are available worldwide, they have their respective user base and the numbers are growing. In order 
for these online marketplaces to become sustainable, it is crucial to look into how they build trust and the way they are ensuring security in their platform so they can protect their users.

Table 1. Global rank of online marketplaces

\begin{tabular}{|l|c|l|}
\hline \multicolumn{1}{|c|}{ International Online Market Place } & Global Rank & \multicolumn{1}{c|}{ Majority of User (\%) } \\
\hline taobao.com & 8 & China $(91.5 \%)$ \\
\hline amazon.com & 10 & United States $(66.9 \%)$ \\
\hline jd.com & 16 & China $(93.3 \%)$ \\
\hline ebay.com & 40 & United States $(54.3 \%)$ \\
\hline alibaba.com & 166 & China $(21.7 \%)$ \\
\hline asos.com & 339 & Japan $(33.6 \%)$ \\
\hline myntra.com & 1052 & India (95.4\%) \\
\hline otto.de & 1084 & Germany (95.8\%) \\
\hline gumtree.com & 1263 & United Kingdom $(83.4 \%)$ \\
\hline rakuten.com & 1874 & France $(44.3 \%)$ \\
\hline
\end{tabular}

*Ranking data from alexa.com/topsites

\section{Criteria of Study}

There are several criteria need to be fulfilled in order to build trust and improve security in online marketplaces. In terms of trust, we are going to discuss on the communication between seller and buyer, review and rating of product, return and refund policy and information sharing. On Security perspective, we will look into Information Security, Protection from Cyber Attacks and Partnership with Third Party. All these criteria will be discussed in specific case studies including Alibaba, Amazon, eBay andTaoBao in the following sections.

\subsection{Communication between seller and buyer}

The nature of online marketplaces is somehow virtual where buyers do not actually meet the seller face-to-face and yet communication place an important role in order to build trust. The buyer often has questions on the product or the seller requires a mechanism to communicate with the seller. Likewise, the buyer also needs to communicate with the seller to enhance the buy-sell relationship. Buyer-seller communication is important to increase the satisfaction in online shopping because it allows customer to understand the product, process of placing order to receive product and eventually increase the satisfaction of the buyer [50]. Quick response to buyer queries, for example through the instant messaging tool, also brings the trust to a higher level as it will show that the seller is committed to clear the uncertainty of the buyer [50] and [51].

\subsection{Review and rating of product, seller and buyer}

Review and Rating of the product are one of the important criteria to build trust in the online marketplace. By having a review and rating product, it will help the buyers to know the details about the product before they plan to purchase. Therefore, it be- 
came one of the best strategies to attract customers and build the experience community. Besides that, the review and rating not only apply to the product. The buyer also can rate the sellers to give clear and transparent details to the public. Through these mechanisms the products establishing transparency will eventually increase the trustworthy among the buyers. There is a study conducted by dimensional research claims that $90 \%$ of online buyers trust their purchasing choices through product review and rating. They still consider the product review and rating especially when deciding to buy expensive products through an online marketplace. This criterion can make or break the trustworthy among buyers in the online marketplace. Thus, the positive feedback and review upon the product can increase more customers in the future. The community interaction is needed in product review and rating to build trust in the online marketplace.

\subsection{Return and refund policy}

It is obvious that great return and refund policy do help to build trust among buyers. A buyer sometimes does have expectations on the product items they bought online, but because there's no physical interaction, the buyer cannot feel and see the item with their own eyes. Buyer can only touch the item they purchased when they open the delivery package and sometimes it just feels different from the picture viewed in the online shop. In this case, flexible and easy return and refund policy will comfort the buyer and lower his dissatisfaction level to the minimum. An easy follow return and refund instructions will further improve the relationship between the seller and buyer [52].

\subsection{Information sharing}

Information Sharing is also important factor to build trust among the buyers when it comes to the online marketplace. The social network allows the buyers to share their thought and experiences about the product to the public. For example, by sharing their opinion through an online forum can provide a guideline to other when making a purchasing decision. Information sharing can reduce uncertainty buyer face regarding the quality of product or sellers. This will help the buyers to communicate the product's quality and also build the trust between buyers and seller in the online marketplace. This mechanism can help the buyers to determine the information reliability and build the trust. There are enormous benefits of information sharing through an online forum for sellers and buyers by gathering the information on customer satisfaction on a product with different communities, price sensitivity, the quality of product and also the changes in attitude of buyers [53]. Using the mechanism of information sharing, buyers can read the review of information shared by communities when making the purchasing decision. Through this, it will increase the trust among the buyers on the product and they will continuously use the online marketplace. Nowadays, buyers explore the details about the product that they are going to purchase through social media or forum rather than asking with their friends or relatives. The buyers will review all the comments about the product before planning to purchase. 
By this way, they will get to know well about the product details more clearly. It will help the buyers to get to know about the quality or the feedback given by the experienced people and hence increase the trust among buyers.

\subsection{Information security}

Encryption algorithms are used to protect the information [54]. Securing the stored information and information transmission isvery important for an online marketplace which needs to build trust among sellers and buyers. It helps to protect data, payment information or emails from access by others or computer hackers. Information is encrypted only by an authorized user. Apart from that, the Secure Electronic Transaction (SET) protocol can be used to provide security of the information. It offers secure and reliable environment for transmitting the credit card information in any online transaction. It can secure customer credit card information from access by third parties. For example, a merchant will transfer transaction coded by the SET to the processing center of the payment card being used and once the transaction is approved, the merchant will also receive the notification from respective bank. Such secured server may help the customer by protecting him against any possible loss of a personal detail. Another technology that is being used for securing the online transactions is Secure Socket Layer or simply "SSL". It offers security for the personal or sensitive information i.e. credit or debit cards or the login information of any user by transferring it in a secure manner. SSL secure the sensitive information from third parties by restricting their access to exchanged information such as credit card numbers and other sensitive information and secures the connection from a server to a browser.

\subsection{Protection from Cyber Attacks}

To ensure the online marketplace is secure, the platform must apply techniques and mechanisms to prevent and stop Cyber Attacks. First of all, the onlinemarketplace must ensure that their website compliance to industrial recognized security standard such as PCI. There's a penalty imposed to merchants who are not compliant with PCI Security Standard. Apart from that, keeping data encrypted is one way to prevent phishing attack. One common method is using SSL to encrypt sensitive data such as credit card and login details. Of course, the users on the online marketplace also need to adopt strong login passwords to help increase the security. Many online marketplaces do provide guidelines in setting up login password.

\subsection{Partnership with Third Party}

Some online marketplaces like Alibaba, eBay and TaoBao partner with the third party to enhance their security level. This partnership will ensure non-manipulation of data by one single party. The third party services will act as monitor to ensure the transactionis completedsecurely. Third party service providers like QualysCloud Platform and Alipayare well known on their security standard where only meeting certain security requirements, they can be chosen as a service partner to the online market- 
place. Furthermore, the third party service providers have to constantly increase or improve the level of security for capturing user's trust from marketplaces where more and more users will continue using online platform for shopping.

\section{Case of Alibaba}

Alibaba is a leading business to business ecommerce company which is situated in China. It offers a marketplace that connects small as well as medium scale sellers and customers throughout the world. The growth of Alibabais astonishing. It started from a general purpose electronic market and transformed into a giant electronic marketplace. While the business was growing rapidly, it faced many challenges and great competition. Presently, ensuring the product and services quality and sustaining a profitable business model are the biggest Alibaba's challenges [18].

By the time of the launch i.e. 1999, marketplaces have formed a community that contains more than 24 million users that are registered with the marketplaces. Till November 2007, the number of users that actually make purchases reached to a record 255,000. Alibaba was launched and was listed on stock exchange of Hong Kong and it raised almost HK $\$ 13.1$ billionin gross proceeds, that makes it the biggest Internet IPO in specifically Asia and generally the second largest worldwide [18].

There are key factors to Alibaba's strategies including trust and payment. Such strategies led Alibaba to its success i.e. strong brand image, offering their customers exceptional value of money, excellent shopping experience, enormous volume of sales and understanding the economies of the scale[18].

Alibaba offers a tool to manage trading which is used by the trading partners in order to communicate in real time. They also have expert teams that are dedicated only for finding new innovations and technologies to catch up with modern trends. Alibaba always try their level best to develop very efficient systems in order to improve the security of online transaction for customers. They introduced "Trustpass" to secure the online transactions. TrustPass issues a certification to the online seller afterverifying their identity and bank account. They also have latest technologies for preventing frauds and spamming. Such technologies can detect fraudulent activities in order to filter some undesirable messages. IT department works continuously to improve accuracy of their systems in order to protect the customers [17].

In 2001, Alibaba introduced a service called TrustPass. For a seller to get a TrustPass certification, they had to get through a third-party identity and bank account verification process. Alibaba also helped sellers create their official-brands and virtual sales fronts. This service marked a breakthrough for the company not only in terms of trust but also in terms of money. On average, certified TrustPass sellers were receiving up to six times more genuine enquires than non-registered sellers. This gave the platform the perfect excuse to start charging small businesses. In Porter Erisman words, 'it made those members still clinging to their free accounts seems less trustworthy. After all, if they had such a good business, why weren'tthey willing to pay up a little to prove it?'. This is how Alibaba transformed its core business from ecommerce to Trust management[56]. 
Alibaba also offers TrustPass Memberships. It is a mechanism for credit enhancing. Members who have gone through authentication and verification procedure can utilize it. Authentication and verification are conducted by a credit agency that is third party and does not belongs to Alibaba. TrustPass offers transparency of identity and legitimacy of the seller between their trading partners. The Information quality is guaranteed for TrustPass membership hence it ensures the confidence of the buy$\operatorname{er}[55]$.

When the ecommerce in China was in emerging state, it was not possible to detect the people that are involved in such transactions due to lack of identity authentication and certificate authority back in 2003. In order to resolve this issue, Alibaba introduced a new platform for the payments called Alipay. It was most suitable for conditions in China. Before the Alipay was introduced, the seller was reluctant to deliver the good to the buyer before he makes payment butAlipay resolved all such concerns and made it easy for the sellers and buyers to safeguard them regarding payment issues.

Alipay was launched by the Alibaba group in 2004. In China, it is now a thirdparty online payment solution.It offers escrow payment method which greatly reduces the risks in online transactions for the customers. Customers only need to release the payment to the seller once they are satisfied with the product. Alipay received license from People's Bank of China in May 2011 which make them a third party payment service provider. Such license enabled them to offer services like mobile payments, online payments, cards for prepaid payment and top up for real name payment accounts[49].

There are many other similar options as Alipay that are involved in ecommerce payments but Alipay is one of the best that really make an impact in people's life by improving them and by turning impossible things into possible.

Following are reasons of Alipay being one of the best [27]:

1. Secured Online Shopping: Alipayowns some leading technologies such as Verisign signed global security certificate, top level data encryption, 128 bit SSL encryption and many security protocols. Such technologies secure the funds of the users in their account and ensures privacy.

2. Unhindered online to offline payments: Alipay has partnership with many banks and other financial institutes so that the customer demand can be fulfilled. They also allow different methods of making payments such as:
(a) Speedy payment
(b) Online transfer through bank
(c) Payments from Account balance
(d) Payments through third parties
(e) Cash on delivery (COD)
(f) Installments on basis of credit cards
(g) Payments through outlets
(h) Card payments through consumption
(i) Credit cards for international payments 
3. Six key values of Alipay: Alipay specifically focuses on meeting the ever changing demands of the customers. Alipay strongly believes on the spirit of innovation and entrepreneurship. In Alipay, we have some notable values with respect to fundamental operations, recruitment, compensation as well as evaluation:

(a) Customer is always first

(b) Working in teams (Teamwork)

(c)

(d) Welcome Change

(e) Integrity or Honesty

(f) Passion in everything you do

(g) Commitment with work

Such amazing cultural values has attracted more and more customers for Alipay as more customers started trusting them for their day to day financial transaction needs. Such values helped Alipay to maintain and boost their company image [27].

Alibaba is a leading ecommerce business in China linking all the small scale as well as medium scale customers and sellers around the world. They started their business with some general electronic marketplace and tuned into huge electronic marketplace. Although today Alibaba is a giant group but in their initial days they also faced many challenges as well as competition. Today, their service quality and profitableness is the biggest defies in Alibaba. The success and popularity of Alibaba is due to its strategy of building trust between users and adopting industry security to ensure transaction integrity. Customer confidence must be gained and strengthened rather than material or technical benefits because gaining trust is the key factor in attracting customers and hence the ability to compete and maximize profits. There are multiple reasons of the growth of TrustPass members in China. The reasons can be divided into external reasons and internal reasons. External reasons include, high internet penetration and the strong economy of China. Such reasons are helping Alibaba's business. Internal reasons include the improvements in the acquisitions of the customers through their reseller networks as well as sales force. Another reason is the introduction of a third party platform for the payments called Alipay. It has partnership with many industrial as well as commercial banks.

\section{Case of Amazon}

Amazon is an online marketplace in the UnitedStates (US) that empowers many third party venders for selling their products. Jeff Bezos is the founder of the Amazon Marketplace. Amazon is $10^{\text {th }}$ ranked in the most visited website in the world according to Alexa. Amazon was started earlier just as an online store for selling books but with the passage of time it has now become a giant that sells almost all kind of products [38].Using the Amazon platform, the third-party sellers increase Amazon's customer base and expands the offers on its site without invest additional inventory.

Amazon has high-quality security measures, and glorious shipping ways that tend to create individuals have a positive experience with the corporate. Users trust Amazon because it has the necessary characteristics that create trust among them. 
Amazon got high marks for meeting customers' expectations for quality according to national most trustworthy brands survey 2017[34].Amazon did not fail to earn their customers trust by providing a more accurate description of the product. Amazon provides plenty of knowledge that many vendors will not. Customers crave for transparency. Amazon provides all positive and negative review of the product to help in their customers' buying decision. They also merged the customer and product intelligence in order to individualize product's experiences so that the count as well as quality of the reviews can be increased or boosted. This consistency and transparency help in earning customers' trust and maintains a longer-term relationship [35].

Besides that, good customer services also increase trustin their marketplace. Amazon has developed a helpful tool for their users' convenience. It shows their worldclass customer service. Amazon also uses the social media in order to interact with customers. Social media is the most powerful tool today in modern world which if used right can really boost ecommerce by addressing the concerns of the customers and develop a strong foundation for fans following.

Amazon is known to have one of the most customer-friendly return policies in the e-commerce world. With easy to read and understand return and refunds terms, Amazon makes it unbelievably simple for customers to return products and get refunds [36].

Amazon hasfive keys on their refund policy such as make return and refund information readily available in the website, offer a quick and simple returns policy, set reasonable refund policies, take full responsibility (even for third-party sellers) and finally think in terms of customer satisfaction (and retention).Amazon focuses on nurturing long-term relationships with customers [36].

The cloud platform or the infrastructure of Amazon is designed in a way that it is extremely available and also scalable because it uses Amazon web services which is also known as AWS. AWS offers industry standards security and their data center is continuously monitored by authorized people only for 24 by 7 . The remote access is assigned according to the managerial and authority level. Their infrastructure is designed as well as managed in a way that meets best practices of security and the wide list of security standards that are found in information technology.

Firewall rules are created by the security concerned authorized group of people in AWS that controls all incoming as well as outgoing traffic. Such rules can actually filters the traffic by using the protocol types. Protocol types that can be used to filter the traffic can be port, TCP, IP address, UDP or ICMP. In order to prevent the DDOS attacks on the network, access control list can be used. Such technique works on the subnet level of the network hence it can be used to blacklist any particular IP address.

The encryption technique on server side can protect the data. There are encryption keys that supports a multi factor encryption, which is strong enough to protect the data. Such keys are known as SSE-S3 and they can actually encrypt every object or item using a combination of keys i.e. a unique key to encrypt object and a master key that keeps rotating. Furthermore, such encryption key also uses very powerful blocks of ciphers and some advanced encryption standards such as a 256bit AES256 in order to encrypt and safeguard the data. 
There are multiple resources in AWS such as VPC, RDS, and EC2 such resources allow for identifying and Access Management helps is creating users, groups, roles, and access level. They can also help to enable the unique credentials required for all users in their provided account. When a user tries to access AWS resource, their username, passwords are authenticated with the use of multifactor authentication technique. In order to grant users, the access to AWS applications and services such as its management console or the APIs, Identity as well as Access Management is utilized. [37].

Table 2. Compare Aws Storage Services In Terms Of Cost, Accessibility and Performance

\begin{tabular}{|l|l|l|l|}
\hline \multicolumn{1}{|c|}{ Services } & \multicolumn{1}{|c|}{ Cost } & \multicolumn{1}{c|}{ Accessibility } & \multicolumn{1}{c|}{ Performance } \\
\hline Amazon S3 & Cheapest for data storage alone & $\begin{array}{l}\text { Accessed from any- } \\
\text { where }\end{array}$ & Slow \\
\hline Amazon EBS & Use-based cost structure & $\begin{array}{l}\text { Only available in a } \\
\text { particular region }\end{array}$ & Faster \\
\hline Amazon EFS & Cost depending on the region & $\begin{array}{l}\text { Can share files be- } \\
\text { tween regions on } \\
\text { multiple EFS instances }\end{array}$ & Faster \\
\hline
\end{tabular}

\section{Case of E-Bay}

After Amazon, eBay is also one of the biggest online marketplace in the world. It allows trading locally as well as internationally. eBay has a very popular and stable customer relationship management system that helps buyers as well as sellers with registration to the eBay. It also helps to collect the users data. eBay has Business to Consumer as well as Consumer to Consumer model which strengthens the customer relationships. Online auctions are the term of eBay [47].

One of the most important factor in online marketplace is to gain the trust of the customer without which online marketplace cannot exists. eBay's ODR Mechanisms is one of the systems which enableusers to trust. Online Dispute Resolution (ODR) helps increate trust among buyers and sellers. ODR is also known as a Feedback System [19]. Buyers or sellers can rate the products and service by providing positive or negative feedback about the products and service. It helps and allows buyers/sellers to review the products as well as the service being provided or transacted [45].

Besides that, eBay also provides independent feedback review which is a thirdparty process [46]. It helps the buyers or sellers to review and evaluate the feedback of specific products or transaction such as motor vehicle sales which is only available for those who buy from the US [30].

eBay is creating trust among buyers and sellers by providing online safety centre [43]. This system helps to avoid conflict among buyers and sellers especially facing when not receiving the item or products order, receiving the wrong item which is not ordered or purchased, receiving the item which is not in good quality such as broken, wrong colour, poor condition, wrong size and more. Besides, eBay allows buyers to returns and refunds the items which do not meet buyer's satisfaction such as broken, does not match the listing description and more [44]. 
eBay Buyer Protection helps buyers and sellers to solve the issues such as buyers not received the purchased items, items are damaged and needs to return and get the refund. Refund will enable the sellers to feel more secure. This policy will ensure consumers satisfaction and trustworthiness [19] and [20].

Security is utmost important to trade in the online marketplace for buyers and seller. eBay provides online payment system which is PayPal. Buyers and sellers allow using PayPal for purchasing and selling the items or products in a safe way by avoiding the fraud. PayPal is the faster and secure way to pay and get the payment via mobile devices. PayPal enables the users to do the payment in a secret way which is without sharing the information and financial credentials to others. On the other hand, PayPal enables global commerce as well [39]. PayPal is the best and suitable method of the payment for eBay. It provides guarantees for buyers as well as its safe, secure and trusted for purchasing good or services via online.

Besides that, for authentication eBay also provides a voluntary idverifier service which will identify and authenticate the identity of buyers and sellers. The ID verification is required for the items/product having more than $\$ 15,000$ price [23].

Just like Amazon, eBay also have some security protocols in hand to secure their customers and vendors. Such solutions are provided by QualysGuard. They selected that specific solution for vulnerability management because Qualys is one of the demands in vulnerability management. QualysGuard plays important roles in eBay by securing the Online Marketplace. It has allowed eBay to do vulnerability assessments, it can also rapidly scan any vulnerability detected anywhere such as in the eBay's network or in the network of their partners [42]. Qualysoffers security of the default market standards. It also has an easy to use web interface which can be used to manage the data related to the security and it is also easy to use on partner networks where their security reputation is highly recommended by more than 8,800 customers who Qualys have been working with.

eBay also has established aprivacy policy [28]. The buyers and sellers of eBay must be eighteen years old or above [21] and [22].Besides that, the users must register to become a member of eBay either to buy or sell by providing their information like name, address, contact details or a valid email. Moreover, users must accept user agreement template as well [32]. eBay also secure the system by tracking the URL, IP address and browser software of the users. It helps to gather or collect bidding information and selling information of the person [31].eBay also hasprovided security center system that allows buyers and sellers to be more secure [21]. It allows the buyers and sellers to access the system when they are facing problems such as fake email, the accounthasbeen hacked,and more by the report to this system. On the other hand, the security center helps users to protect their account information, devices and also help to stay safe from hackers or scammers [40]. 


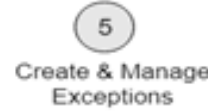

Exceptions
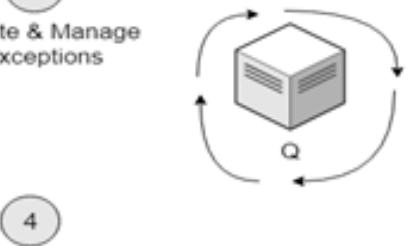

Create Compliance Policy Reports (Per host, asset group)

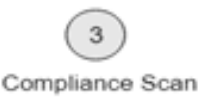

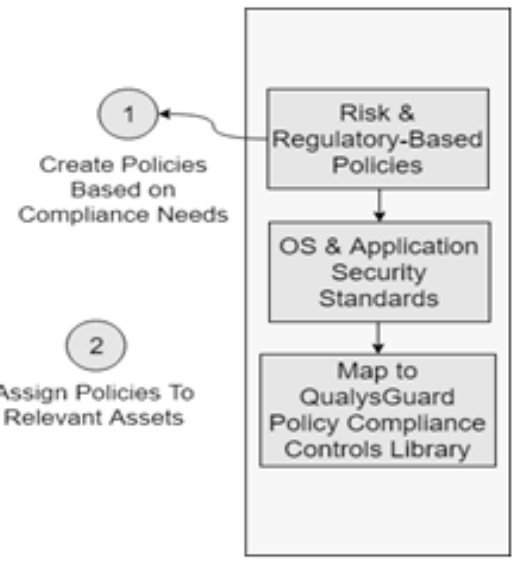

Fig. 1. Automated Workflow or the policy compliance life cycle using Qualys Guard's policy compliance

On top of that, eBay provides a tracking system for buyers which helps and ease the buyers to check the current location of the items or estimate when their orders will be received by entering the tracking number of the purchased items or products[41].

\section{Case of Taobao}

Taobao is an online marketplace in China to promote consumer-to-consumer (C2C) online trading, which was founded by its mother company, Alibaba in the year 2003. Taobao is another one of the biggest ecommerce giant in the world and also ranked the eight most visited website globally according to Alexa. Over 600 million active users are actively doing business on Taobao. Taobao instant success overthrown eBay in China, which was the top $\mathrm{C} 2 \mathrm{C}$ online marketplace in China before the commencement of TaoBaoand its operation was seized in the year 2006 after just three years of operation of TaoBao[24]. TaoBao enormous success and popularity is highly credited to its strategy in building trust among users and adopting industry security level to ensure transactions are safe.

As discussed before, building user's trust is very important so for achieving that, TaoBao has adopted two mechanisms to increase trust namely Institutional Based and Social Based. Evidence shows that TaoBao is significantly applying four main features in Institutional Based mechanism, i.e. Reputation System, Escrow Services, Credit Card Guarantees and Trusted Intermediary [25].

Due to the nature of virtual anonymity of online marketplace (buyer do not meet seller face to face), there's a need of a system to ensure the seller can be trusted in order to build trust among the buyers. TaoBao adopted reputation system to give a rate or score to the online seller's implying their reputation. Buyers can give scores to the buyers they were dealing with in the past. Furthermore, they can give review or feedback which publish online as a reference to other interested buyers. Even though 
there's no face to face meeting between buyer and seller, this reputation system will review the online seller reputation, and with the addition of real user feedback, it provides a sufficient level of guidelines and hence increase the trust on dealing the seller.

Escrow service such as Alipay is very popular among the users in TaoBao whereby the payment will only release to the seller when the buyer confirms the goods received are genuine and meet the expectation. Figure 2. shows the payment flow using Alipay in TaoBao online marketplace. This feature boosts the confidence level of buyers to shop on TaoBao as there are too many fraud cases regarding fake products were reported. Credit card companies who partner with TaoBao guarantee a secure channel for online payment where they offer buyers zero liability if there is fraud involved in an online transaction. These credit card company verified online seller strictly regarding security capability before accepting them as a business partner.

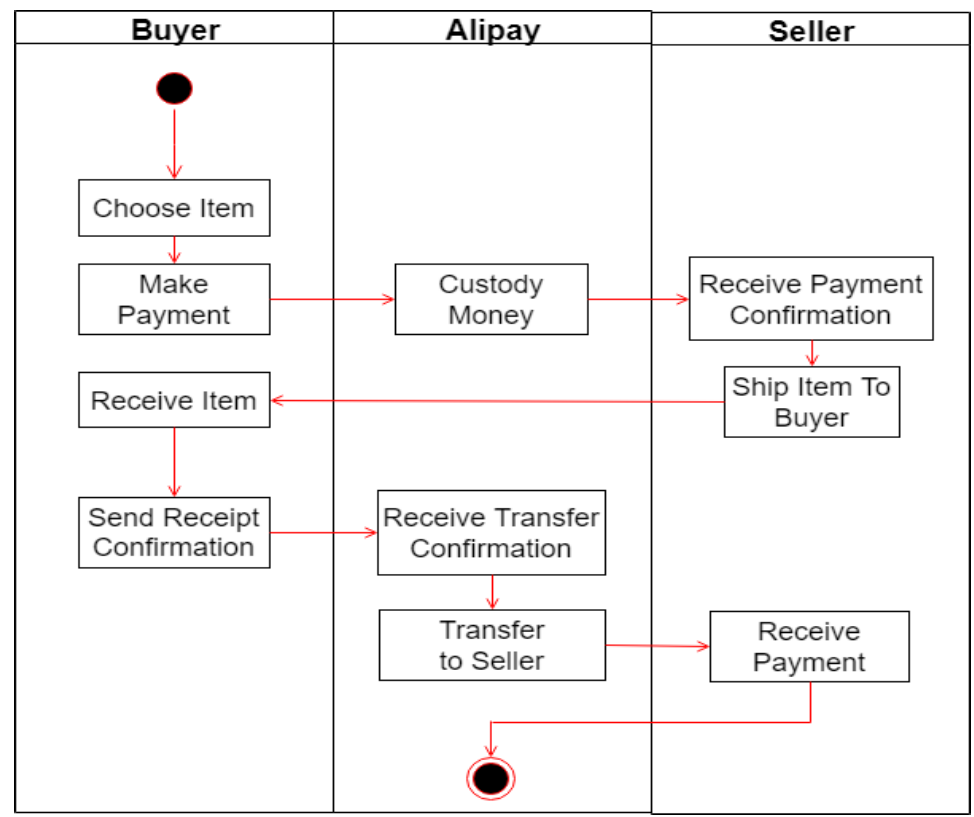

Fig. 2. Payment flow using Alipay in TaoBao Online Marketplace [27]

Lastly, TaoBao also ensures trusted intermediary to enforce fair rules, guidelines, and procedures to avoid dispute among buyers and sellers. Those rules avoid sellers or buyers to take advantage of each other. Hence, it helps to build customer satisfaction among the user.

Apart from institutional-based mechanism to enhance trust, the social mechanism is also considered an important factor to help increase the level of trust. A very crucial feature in TaoBao that customers like to use is Instant Messaging. Customers like to get instant feedback from the online seller. It is proven that a prompt reply from the seller helps facilitate the satisfaction among buyers. TaoBaois very well known of the 
online seller that give prompt response to the questions from the buyer. Furthermore, TaoBao platform message box is almost like email. It enables the buyer to leave a message to the seller especially those from overseas that are located at a different time zone to the seller. The seller can reply to the queries from buyer to clear their doubt. Both instants messaging and message box inculcates communication between the buyer and seller that compensate the drawbacks of no face-to-face meeting between them.

Another social feature that TaoBao frequently promotes is its online community forum. Active users of TaoBao may share their experience for example; how to prevent fraud is found very useful and encouraging for the users. Based on those experience sharing, users can have an idea or guideline to prevent unhappy incidents.

Security is no doubt the fundamental issue of online marketplace [26]. For the world's largest e-commerce website, security is a must to ensure a safe environment that helps to promote their services worldwide. According to a comparison survey of 120 respondents, 65 respondents strongly agree that TaoBao has better security than JingDong also known as JD.com [24]. According to data disclosed by Alibaba, more than half of TaoBao users adopt Alipay as the payment or escrow service.

As discussed before, Alipay is an extremely trusted and well-known system for the online payments being used in different parts of China where their security level is known for the best. Alipay, a third-party payment system company founded by Alibaba, disclose the six major security settings that incorporated in Alipay[27].

First, Alipay Security Controls ensure compatibility with the mainstream operating system in the market and use multipleencryptionsby SSL encrypted transmission that protectskey personal information like login password. Next, in order to change key information of Alipay account or complete payment on $\mathrm{PC}$, there is a need to verify the contact number via SMS in order to complete the required task.

Alipay issue Digital Certificate to the devices of their eligible users. In order to fully use the service of Alipay, the devices that are used by the users must have the latest digital certificate. If any evil guy steals the login password, they cannot perform any transaction by logging in the Alipay account with the stolen password if they use other devices that don't have the legal digital certificate. Another powerful security tool used by Alipay is Payment Shield. Payment Shield makes sure that the information that is being transmitted on the network while a user is making some transaction is secure and confidential. This tool enables protection of personal data related to Alipay account.

To further ensure an international level of security feature, Alipay joins together with China Unicom to launch a service called Bao Order. In a nutshell, this service generates a random six-digit numbers whenever user intend to pay using Alipay through his account balance or through some credit card. Without that six-digit codes, there's no way to complete the transaction.

Last but not least, another security feature used in Alipay is Security Protection Question where Alipay user can retrieve his or her password if they were stolen or forgot about it. Unfortunately, this feature only allows using a PC platform.

TaoBao as the world's largest e-commerce C2C platform in the world, trust among the users and security features are the most important aspects for them to create a 
sustainable and growing platform so that they can stay competitive in the online shopping market. Although TaoBao already uses many creative tools to promote trust among users, it is still not enough to cater to their demands, and more efforts need to be carried out to make it better. On top of that, TaoBao never stops to enhance their efforts to ensure security environment, many sophisticated and high level features are already being introduced in Alipay. It is never enough as there is always new cyber threats trying to jeopardize its smooth function. Nevertheless, TaoBao is still the favourite $\mathrm{C} 2 \mathrm{C}$ online marketplace in China.

\section{Discussion}

This paper discusses how online marketplaces, in particular, build trust among users and create a secure environment for them. Four popular online marketplaces were analyzed,i.e. Alibaba, Amazon, eBay, and TaoBao.

From the findings, all the four online marketplaces adopted similar methods or techniques to build trust among their respective users. One important and popular tool used is product review and feedback system. Alibaba, Amazon, eBay, and TaoBao supports the principle of transparency regarding product review, i.e. they allow public to view positive and negative review on a specific product. This method enables buyers as well as sellers to have a better understanding of the product.

From the articles review, findings show that Amazon and eBay emphasis on product return and monetary refund if the item does not meet buyer's expectation. Both of the online platformsmake the return and refund policy clear on their website and such policy is easy to understand and follow. On the other hand, Alibaba and TaoBao focus more on customer experience rather than put more effort on return and refund policy.

Other tools that help to build trust are also implemented in those four online marketplaces such as Instant Messaging, Message Box and Online forum to provide more ways for the buyers to interact with sellers as online marketplaces do not enable faceto-face meeting.

Despite adopting their unique security techniques, Alibaba, Amazon, eBay, and TaoBao have one common goal i.e., to ensure a safe and secure online environment at the same time and to protect user privacy.

Amazon launches the online marketplace using theirdeveloped cloud platform,i.e. AWS where the security of the data center is monitored 24/7. They create special firewall that controls the incoming and outgoing network traffic. Furthermore, they also implement Access Control List (ACL) for their employee based on group and roles to control access to their main service. Amazon also enhances security by using advanced encryption standard (AES-256) and using the SSE-S3 encryption key to ensure server-side encryption. These security features ensure Amazon stays the popular online marketplace in the world.

On the hand, eBay adopted PayPal as their primary payment service to ensure payment security. PayPal is well known as they adopted the world-class security control. Also, eBay uses Qualys Guard to perform regular vulnerability scan on their platform. This is one of the health check technique to enhance its security. eBay also 
tracks their user's URL, IP and browsers to avoid fraud. Lastly, eBay establishes Security Center for their users to report any fraud case.

Alibaba and TaoBao adopted Alipay as their primary payment system. Alipay enhances security with several features. First, it adopts multipleencryption methodsbased on SSL encryption to protect user's data. Secondly, it offers Security Controls to ensure the application is compatible with a mainstream operating system to avoid cyber-attacks. Alipay issues Digital Certification to the devices that install the application, without that certification, the user cannot perform any transaction.

In order to modify user account detail, Security Adoption Questions is required to be answered. Last but not least, Alipay partnered with Unicom to launch Bao Order where Alipay users' six digit numbers are sent to users for any transaction is performed. This feature helps to prevent fraud happening among TaoBao users.

Table 3. Summary of online marketplaces (alibaba, amazon, ebay and taobao) trust and security methods

\begin{tabular}{|c|c|}
\hline \multicolumn{2}{|c|}{ Alibaba } \\
\hline Methods Building Trust & Methods Enhancing Security \\
\hline $\begin{array}{l}\text { Strong Branding. } \\
\text { Valueadded electronic marketplace. } \\
\text { Excellentshopping experience. } \\
\text { Trade Manager Tool. } \\
\text { PPC advertising }\end{array}$ & $\begin{array}{l}\text { Alipay as the primary payment system. } \\
\text { TrustPass credit certification }\end{array}$ \\
\hline \multicolumn{2}{|l|}{ Amazon } \\
\hline Methods Building Trust & Methods Enhancing Security \\
\hline $\begin{array}{l}\text { Transparent product review. } \\
\text { Social media for the buyer to interact with the } \\
\text { seller. } \\
\text { Friendly return policy. }\end{array}$ & $\begin{array}{l}\text { AWS cloud platform (monitor 24/7) } \\
\text { Access Control List } \\
\text { Advanced encryption with AES-256 } \\
\text { SSE-S3 encryption keys } \\
\text { Identity and Access Management }\end{array}$ \\
\hline \multicolumn{2}{|l|}{ eBay } \\
\hline Methods Building Trust & Methods Enhancing Security \\
\hline $\begin{array}{l}\text { Online Dispute Resolution (ODR) Sellers and buyers' } \\
\text { rating } \\
\text { Independent Feedback Review by a third party } \\
\text { Online Safety Center } \\
\text { Easy return and refund } \\
\text { Buyer Protection policy }\end{array}$ & $\begin{array}{l}\text { PayPal as the primary payment system. } \\
\text { Adopt QualysGuard as vulnerability assessment tool } \\
\text { Tracks users' URL, IP, and browsers. } \\
\text { Security Center for users to report any fraud. }\end{array}$ \\
\hline \multicolumn{2}{|l|}{ TaoBao } \\
\hline Methods Building Trust & Methods Enhancing Security \\
\hline $\begin{array}{l}\text { Institutional Based: } \\
\text { Reputation System } \\
\text { Escrow Service } \\
\text { Credit Card Guarantees } \\
\text { Trusted Intermediaries } \\
\text { Social Based } \\
\text { Instant Messaging } \\
\text { Message Box } \\
\text { Online Community Forum }\end{array}$ & $\begin{array}{l}\text { Alipay as the primary payment system: } \\
\text { Multiple encryption based on SSL encryption. } \\
\text { Security Controls } \\
\text { Digital Certificate for devices } \\
\text { Partner with Unicom to launch Bao Order } \\
\text { Security Adoption } \\
\text { Questions }\end{array}$ \\
\hline
\end{tabular}




\section{Conclusion}

Online marketplaces have become incredibly popular nowadays, and online shopping already integrates into our daily lives as the number of online shoppers increase exponentially for the last decade.

Alibaba, Amazon, eBay, and TaoBao are the most famous online marketplaces in the world and more and more users subscript to them. The increase of transaction in these online marketplaces are mainly due to the users have trust on them, and also they think that it is secure to purchase goods through these online marketplaces.

This review paper tries to shed light on the trust-building mechanism the online marketplaces adopt respectively. From the findings, Alibaba, Amazon, eBay, and TaoBaouse similar tools to promote trust,i.e. to create better communication between buyer and seller. Due to the nature of virtual anonymity, the bridge to enable communication such as Instant Messaging or message box are crucial.

Furthermore, in order to build trust among buyers, the review mechanism is necessary to provide feedback on the product. Social based mechanism also has a very important part in gaining the trust. The clear example is a forum where frequent users can share their experience with the new subscribers.

On top of that, the security aspect also is the focal point for online marketplaces. Alibaba, Amazon, eBay and TaoBao have each of their unique techniques to ensure security and privacy, but mainly they are adopting encryption to reach their goals. No doubt encryption is the best method to create a secure platform, especially for online marketplaces. Additional methods like access control and management and third-party payment systems like PayPal and Alipay are also adopted by the mentioned online marketplaces.

Although Alibaba, Amazon, eBay, and TaoBao already implement various worldclass techniques to create trust and enhance security, it is always not enough to cater the innovative and notorious cyber-threats. A new method and more robust techniques must always be invented to handle such threat in order for the online marketplace to sustain their operation. Hence, this paper provides an insight into future research on the current methods and techniques used by the mentioned online marketplaces to ensure trust and security. There is still more research needed so that the trust and security among the online marketplaces can be increased further.

\section{Acknowledgement}

This research was funded by the Fundamental Research Grant (13144). The authors would like to thank Universiti Utara Malaysia and Ministry of Education Malaysia for funding this research. 


\section{References}

[1] Michael Luca Harvard Business School 2016 Designing Online Marketplaces: Trustand Reputation Mechanisms

[2] Patrick McCole(2010) Trust considerations on attitudes towards online purchasing: The moderating effect of privacy and security concerns

[3] D. Gefen. Reflections on the dimensions of trust and trustworthiness among online consumers ACMSIGMIS Database, 33 (3) (2002), pp. 38-53. https://doi.org/10. $\underline{1145 / 569905.569910}$

[4] S.L. Jarvenpaa, N. Tractinsky, L. Saarinen, M. Vitale. Consumer trust in an Internet store: a cross- culturalvalidation. Journal of Computer Mediated Communication, 5 (2) (1999). https://doi.org/10.1111/j.1083-6101.1999.tb00337.x

[5] D.J. Kim, Y.I. Song, S.B. Braynov, H.R. Rao. A multi-dimensional trust formation model in B-to-ecommerce: A Conceptual Framework And Content Analyses Of Academia/Practitioner Perspective. Decision Support Systems, 40 (2) (2005), pp. 143-165. https://doi.org/10.1016/i.dss.2004.01.006

[6] P. Grabosky. The nature of trust online. The Age (2001), pp. 1-12.

[7] Fung, R. and Lee, M. (1999), "EC-trust (trust in e-commerce): exploring the antecedent factors",Proceedings of the 5th Americas Conference on Information Systems.

[8] Chellappa, R.K. (2001), "The role of perceived privacy and perceived security in the development of trustin electronic commerce transactions ${ }^{\text {eeee }}$,ebizlab working paper, Marshall School of Business, University of South California, Los Angeles, CA.

[9] Graves, P., and M. Curtin. 2000. Bank One Online Puts Customer Account Information At Risk. http://www.interhack.net/pubs/bankone-online.

[10] Neyses, J. 2002, Higher Education Security Alert From the U.S. Secret Service: List of Keystroke Logging Programs. http://www.unh.edu/tcs/reports/sshesa.html.

[11] Adams, C., and S. Farrell. 1999. Internet X.509 Public Key Infrastructure Certificate Management Protocols. Internet RFC 2510.

[12] Denale, R., Liu, X., And Weidenhamer, D. Quarterly e-commerce retail sales, 2nd quarter 2015. U.S. Census Bureau.

[13] Michael Luca Harvard Business School 2017

[14] Ms. Palak Gupta(2016) E-Commerce- Study of Privacy, Trust and Security from Consumer's Perspective.

[15] Best, R. J. (2013). Market-based management, strategies for growing customer value and profitability. New Jersey: Pearson Education, Inc

[16] Essays, UK. (November 2013). Market Analysis And Strategy For Alibaba Marketing Essay. Retrieved from http://www.ukessays.com/essays/marketing/market-analysis-andstrategy-for-alibaba-marketingessay.php?cref $=1$

[17] Yazdanifard, R., \& Li, M. T. H. (2014). The Review of Alibaba's Online Business Marketing Strategies, which Navigate them to Present Success. Global Journal of Management And Business Research.

[18] Qing, H. H., \& Xue, Z. S. (2009, September). A model for value-added E-marketplace provisioning: Case study from Alibaba. com. In Conference on e-Business, e-Services and e-Society (pp. 65-72). Springer, Berlin, Heidelberg. 
[19] Resnick, P., and R. Zeckhauser. 2001. Trust among strangers in Internart transactions: Empirical analysis of eBay's reputation system.Working Paper for NBER Workshop on Empirical Studies of Electronic Commerce.

[20] http://www.si.umich.edu/ presnick/papers/ebayNBER/index.html.

[21] Gao, T., Sirgy, M.J., and Bird, M.M. "Reducing Buyer Decision-Making Uncertainty in Organizational Purchasing: Can Supplier Trust, Commitment, and Dependence Help?," Journal of Business Research (58:4), 2005, pp. 397-405.

[22] Jarvenpaa, S.L., and Tractinsky, N. "Consumer Trust in an Internet Store: A Cross-Cultural Validation," Journal of Computer Mediated Communication (5:2), 1999, pp. 1-35.

[23] Pavlou, P., and Gefen, D. "Building Effective Online Marketplaces with Institution-based Trust," Information Systems Research (15:1), 2004, pp. 37-59.

[24] Guidera, J. 2000. FTC, states target online-auction fraud. Wall Street Journal (Eastern Edition). February 15. p.B8.

[25] Y. Zhang, "Customer Satisfaction Comparison between Jingdong and Taobao," International Journal of Science and Business, vol. 1, pp. 149-157, 2017

[26] C. Ou, W. Wong, R. Davison, "Beyond Institution-Based Trust: Building Effective Online Marketplaces with Social Mechanisms," International Conference on Information Systems 2010, Paper 207. http://aisel.aisnet.org/icis2010_submissions/207

[27] S.A. Jinyevu, N. A. Mwasha, "Exploring Foreigners' Online Shopping Experience in China: A Case of Taobao," European Journal of Business and Management, vol. 6, no. 5, 2014

[28] R. Liu, "The Role of Alipay in China”, Nijmegen, Radboud University, Nijmegen, The Netherlands, retrieved from:www.ru.nl/publish/pages/769526/z01_rongbing_liu.pdf

[29] Ray, T. 2000. Trust in big business. Smartmoney.com. June 27. www.smartmoney.com/smt/columns/tech/index.cfm?story=200006271.

[30] Cox, B., and Olavsrud, T. "eBay Bans Nazi, Hate Group Memorabilia," (2001:May 3), 2001, http://www.internetnews.com/ec-news/article.php/758221

[31] Dennehy, M. 2000. eBay motors breakdown? AuctionWatch.com. June 23.

[32] Porter, M.E. 2001. Strategy and the Internet. Harvard Business Review. March: $63-78$.

[33] Roth, D. 2000. Fraud's booming in online auctions, but help is here. Fortune. May 29. p.276.

[34] Wolverton, T. "eBay riding Net auction industry's wave,"), 2001,

[35] http://news.com.com/2100-1017-269211.html?legacy=cnet_

[36] https://www.retaildive.com/news/study-amazon-tops-in-consumer-trust-and-joy/449221/

[37] https://www.forbes.com/sites/anthonysmith/2017/11/16/what-amazon-key-teaches-usabout-trust-in-customer-relationships/\#35e9938577d0

[38] https://herothemes.com/blog/what-you-can-learn-amazons-return-policy/

[39] https://cloudacademy.com/blog/amazon-web-services-security/

[40] https://en.wikipedia.org/wiki/Amazon_(company)

[41] https://dzone.com/articles/confused-by-aws-storage-options-s3-ebs-amp-efs-explained

[42] https://pages.ebay.com/securitycenter/index.html

[43] https://www.ebay.com/help/buying/shipping-delivery/tracking-item?id=4027

[44] https://www.qualys.com/customers/success-stories/ebay-securing-worlds-onlinemarketplace-qualysguard/

[45] http://pages.ebay.co.uk/safetycentre/

[46] https://www.ebay.com/help/returns-refunds 
[47] http://pages.ebay.com/help/feedback/index.html

[48] eBay.com/Motors

[49] Houser, Daniel and John Wooders. 2000."Reputation in Auctions: Theory, and Evidence from eBay".

[50] Privacy, Integrity and Security at eBay. Working paper, Yale University.

[51] Lin, R., \& Xie, J. (2014). Understanding the adoption of third-party online payment: An empirical study of user acceptance of Alipay in China.

[52] Hu, M., Rabinovich, E. \& Hou, H. (2015). Customers Complaints In Online Shopping: The Role of Signal Credibility. Journal of Electronic Commerce Research, Vol. 16, No. 2.

[53] Guo, S., Wang, M. \& Leskovec, J. (2011). The Role of Social Network in Online Shopping Information Passing, Price of Trust, and Consumer Choice.

[54] N Raunaque., MA Imam \& MA Raja. (2016). E-Commerce - Customer Experience and Relationship. International Journal of Advanced Engineering, Management and Science (IJAEMS), Vol. 2, Issue. 7.

[55] Dennis A.Pitta and Danielle Fowler "An untapped resource for consumer marketers", "Journal of Consumer Marketing”, 2016, pp. 266-269

[56] Thomas, L., Xiaodong, D.: Building online trust through privacy practices, International Journal of Information Security, Vol. 6, No. 5, 2007, pp. 323-331. https://oi.org/10. $\underline{1007 / s 10207-007-0028-8}$

[57] Fu, C., \& Huang, L. (2010, August). A study of B2B e-service development in China: eservice capability and customer segmentation perspective. In Proceedings of the 12th International Conference on Electronic Commerce: Roadmap for the Future of Electronic Business (pp. 189-199). ACM. https://doi.org/10.1145/2389376.2389402

[58] Bergero, B. S. (2018). Design for trust. The role of trust in platforms enabling peer-to-peer interactions.

\section{Authors}

Osman Ghazali is an Associate Professor and Deputy Dean of the School of Computing, Universiti Utara Malaysia. Osman holds a Ph.D. degree in Information Technology (Networking) from Awang Had Salleh Graduate School, Universiti Utara Malaysia (AHSGS). He did his post-doctoral as a research scientist at the School of Engineering \& Applied Science, Aston University (EAS) in 2012. In 2011, Osman was the Head of Computer Science Department, School of Computing, Universiti Utara Malaysia. Before that, from 2009 to 2011, he was the Technical Chairperson at the University Teaching and Learning Center, Universiti Utara Malaysia. Dr. Osman has more than 100 publications as refereed book chapters and refereed technical papers in journals and conferences. He is a senior member of the InterNetworks Research Laboratory. He is also a member of the IEEE and the ACM.

Chun Yang Leow received his Bachelor Degree in Manufacturing Engineering with Management in 2010 from UniversitiSains Malaysia. He has been working as Process Engineer for Keysight Technology and Sample Build Engineer for Robert Bosch (M) SdnBhd Penang. Currently, he is a Master student in Information Technol- 
ogy in Universiti Utara Malaysia, Malaysia. His research interests are Cyber Security and Cryptocurrency.

Shahzad Qaiser received his bachelor degree in Computer Science in 2017 from BahriaUniversitiy, Karachi, Pakistan. Currently, he is a Master's Information Technology student at Universiti Utara Malaysia, Malaysia where he scored 4 out of 4 CGPA in consecutive two semesters. His research interests are Data Mining, Sentiment Analysis, Machine Learning, Artificial Intelligence, and Cloud Computing.

NanthiniPattabiraman completed a bachelor degree in Computer Science in Software Engineering (BSc.SE) in 2017 from UniversitiTeknologi Malaysia (UTM). Currently, she is pursuinga master in Information Technology (MSc.IT) in Universiti Utara Malaysia (UUM).

Sathiyaroobaa Vasuthevan received her bachelor degree in Computer Science in 2016 from Universiti Selangor. Currently, she is a master student at University Utara Malaysia, Malaysia.She completed her STPM at Sekolah Menengah Kebangsaan Tunku Abdul Rahman, Nibong Tebal Pulau Pinang in 2012.

Eman Mohamed Abdusalam received her bachelor degree in Computer Science in 2006 fromTripoli College of Science and Technology, Tripoli, Libya.She previously worked as an intern at Tripoli College of Science and Technology from 2007 to 2016.She also worked as a research supervisor from 2009 to 2011.Currently, she is a master studentat Universiti Utara Malaysia, Malaysia. Her research interests are Information Technology

Mustafa M. Barakat received his bachelor degree in Computer Science in 2005 from Al-Mamon University, Baghdad, Iraq. He is a master student at School of Computing in Universiti Utara Malaysia, Malaysia. His research interests are cloud computing, network and distributed system security, human computer interaction and usability evaluation.

Article submitted 2019-01-12. Resubmitted 2019-02-25. Final acceptance 2019-02-25. Final version published as submitted by the authors. 